\title{
EFFECT OF PLANTING DATE AND FOLIAR SPRAY WITH POTASSIUM SILICATE ON GROWTH AND YIELD OF SOME GARLIC CULTIVARS
}

\author{
Yaser A.E. Mohamed, E.A. El-Ghamriny, A. Bardisi" and Dalia A.S. Nawar \\ Hort. Dept., Fac. Agric., Zagazig Univ., Egypt
}

Received: 07/02/2019 ; Accepted: 24/02/2019

\begin{abstract}
A field experiment was carried out during the two successive winter seasons of 2016/2017 and 2017/2018 in a private Farm at Hehia Distrect, Sharkia Governorate, Egypt, to study the effect of planting date, and foliar spray with potassium silicate on dry weight, bulb yield and its components of Balady and Sids 40 garlic cultivars under clay soil conditions and using flood irrigation system. The obtained results indicated that planting Balady cultivar on $1^{\text {st }}$ Oct. and spraying with potassium silicate ( $\mathrm{K}$ silicate) at $2 \mathrm{ml} / 1$ increased dry weight of leaves/plant, bulb and total dry weight/plant, yield of grades 1, 2, 3 and 4, exportable, marketable and total yield/fad., as well as average bulb weight.
\end{abstract}

Key words: Garlic, planting dates, cultivars, potassium silicate, dry weight, yield and its components.

\section{INTRODUCTION}

Garlic (Allium sativum L.), a member of the Alliaceae family, is one of the most aromatic herbaceous annual spices (Kurian, 1995). It is the second most widely spice crop of the cultivated Allium crops, next to onion in the world (Purseglove, 1975) with a characteristic pungent smell. In Egypt, the total cultivated area of garlic, was about 29688 fad., during 2016 season which produced 280216 tons with average of 9.438 tons/faddan (FAO, 2016).

Planting dates plays an important role on the growth and yield of garlic. Garlic is known to be thermo and photo-sensitive crop (Jones and Mann, 1963) and its vegetative growth and bulb formation are greatly influenced by growing environment, such as cool weather and grows well in a well-drained soils (Rahim and Fordham, 1988).

There were significant differences among planting dates of garlic $\left(15^{\text {th }}\right.$ Sept., $1^{\text {st }}$ Oct. and $15^{\text {th }}$ Oct.) concerning dry weight (Abdalla et al., 2011; Gunda, 2013; Vidya et al., 2013; Hassan et al., 2016) on garlic, and yield and its components (Muhammad et al., 2001; Ahmed,

\footnotetext{
* Corresponding author: Tel. : +201271887153

E-mail address: abdallabardisi@yahoo.com
}

2002; Bhuiya et al., 2003; Rahim et al., 2003; El-Zohiri and Farag, 2014; Youssef and Tony, 2014; Choudhary, 2015; Mohammad, 2018).

Silicon $(\mathrm{Si})$ is the second most abundant element in the earth crust, yet its role in plant physiology has been poorly understood and attempts to associate $\mathrm{Si}$ with metabolic or physiological activities have been inconclusive. Although $\mathrm{Si}$ has not been classified as an essential element for higher plants, it has been shown to be beneficial for plant growth (Epstein, 1994 and 1999). Silicon has been shown to ameliorate the adverse effects of heavy metals on plants.

Foliar spray with potassium silicate increased plant growth, and yield and its components on strawberries (Wand and Galletta 1998), bean (Abou-Baker et al., 2011), sweet pepper (Kamal, 2013), potato (Salim et al., 2014), Jerusalem artichoke (Abou El-Khair and Mohsen, 2016) and pea (Elrys and Merwad, 2017).

Therefore, the aim of this work was to determin suitable planting date and potassium silicate rate to obtain high growth and best productivity of two garlic cultivars under clay soil and using flood irrigation system. 


\section{MATERIALS AND METHODS}

A field experiment was carried out during the two successive winter seasons of 2016/2017 and 2017/2018 in a private Farm at Hehia Distrect, Sharkia Governorate, Egypt, to study the effect of planting date and foliar spray with potassium silicate on growth, yield and its components of some garlic cultivars (Balady and Sids 40) under clay soil conditions and using flood irrigation system.

The physical and chemical analyses of the soil are presented in Table 1.

This experiment was included 18 treatments, which were the combinations among three planting dates $\left(15^{\text {th }}\right.$ Sep., $1^{\text {st }}$ Oct. and $15^{\text {th }}$ Oct.), two cultivars (Balady and Sids 40) and three levels of potassium silicate $(0,1$ and $2 \mathrm{ml} / \mathrm{l})$. These treatments were arranged in a split - split plot design with three replications. Planting dates were randomly arranged in the main plots, cultivars were randomly distributed in the sub plots and potassium silicate rates were randomly arranged in the sub sub plot.

Garlic plants were sprayed with $\mathrm{K}$ silicate rate three times at 60,80 and 100 days after planting. The experimental unit area was $10.8 \mathrm{~m}^{2}$. It contained three ridges with $6 \mathrm{~m}$ length and $60 \mathrm{~cm}$ in width. One ridge was used for the samples to measure vegetative growth and the other two ridges were used for yield determination.

All plots received equal amounts of farmyard manure (FYM) at $30 \mathrm{~m}^{3} / \mathrm{fad}$., and 54,54 and $76.5 \mathrm{~kg} /$ fad., of $\mathrm{N}, \mathrm{P}$ and $\mathrm{K}_{2} \mathrm{O}$, respectively in the form of ammonium sulphate $(20.6 \% \mathrm{~N})$, calcium superphosphate $\left(\begin{array}{llll}15.5 & \% & \mathrm{P}_{2} \mathrm{O}_{5}\end{array}\right)$ and potassium suphate $\left(50 \% \mathrm{~K}_{2} \mathrm{O}\right)$. One third of mineral fertilizers were added during soil preparation with the same rate of FYM and the rest amounts were added at three portions as soil application monthly beginning one month after planting. The normal agricultural practices were carried out as commonly followed in district.

\section{Data Recorded}

\section{Growth parameters}

Ten plants from each experimental unit were randomly taken at 105 and 135 days after planting (DAP) in both seasons and the following data were recorded:

\section{Dry weight}

The different parts of garlic plant; i.e., bulb and leaves were oven dried at $70^{\circ} \mathrm{C}$ till constant weight, and then the following data were recorded: Bulb dry weight/plant (g), leaf dry weight/plant (g), and total dry weight (bulb + leaves)/ plant (g).

\section{Yield and its components}

Bulbs of every plot were harvested at proper maturity stage of bulbs (200 DAP approximately), then translocated to a shady place in the same day for curing. Plants were placed for about two weeks in a shady place at $25 \pm 5^{\circ} \mathrm{C}$ and $60-75 \%$ $\mathrm{RH}$, and then graded into four categories according to the Ministry of Economic for Garlic Exportation (1963) as follows: Grade 1: Bulbs with diameter above $5.5 \mathrm{~cm}$, Grade 2: bulbs with diameter between $4.5-5.5 \mathrm{~cm}$. Grade 3: Bulbs with diameter between 3.5-4.4 cm, and Grade 4: Bulbs with diameter less than $3.5 \mathrm{~cm}$.

Then after, each grade was weighed separately in the same day and the following data were recorded: Exportable yield (grade $1+$ grade 2 ) ton/fad., marketable yield (grade $1+$ grade $2+$ grade 3 ) ton/fad., total yield (grade $1+$ grade $2+$ grade $3+$ grade 4 ) ton/fad., and average bulb fresh weight was measured.

\section{Statistical Analysis}

The data was subjected to proper statistical analysis of variance according to Snedecor and Cochran (1980) and means separation were done according to least significant differences (LSD) at 5\% level.

\section{RESULTS AND DISCUSSION}

\section{Dry Weight}

\section{Effect of planting date}

Results in Table 2 show that there were significant differences among planting dates with respect to dry weight of leaves, bulb and total dry weight/plant at 105 and 135 days after planting (DAP) in both seasons. At 135 DAP, planting on $1^{\text {st }}$ Oct. increased dry weight of leaves, bulb and total dry weight/plant in both seasons compared to other planting dates $\left(15^{\text {th }}\right.$ Sep. and $15^{\text {th }}$ Oct.). 
Table 1. The physical and chemical properties of soil during 2016/2017 and 2017/2018 (average of two seasons)

Character

Soil particles distribution

Sand (\%)

Silt (\%)

Clay (\%)

Texture

Field capacity (FC) (\%)

$\mathrm{CaCO}_{3}(\%)$

Organic matter (\%)

$\mathbf{p H}^{*}$

$\operatorname{EC}\left(\mathrm{dSm}^{-1}\right)^{* *}$

Soluble cations and anions (meq/100 $\left.\mathrm{g}^{-1}\right)$

$\mathrm{Ca}^{++}$

$\mathbf{M g}^{++}$

$\mathrm{Na}^{+}$

$\mathbf{K}^{+}$

$\mathrm{CO}_{3}{ }^{=}$

$\mathrm{HCO}_{3}^{-}$

$\mathrm{Cl}^{-}$

$\mathrm{SO}_{4}=$

Available nutrients (mg/ $\mathrm{kg}^{-1}$ soil)

Available N

Available P

Available K
78.4

Value

22.07

35.03

42.90

Clay loam

25.4

1.17

1.23

1.18

1.64

2.09

0.18

0.00

0.76

1.69

4.02

172

"Soil water suspension 1:2.5 "soil water extract 1:5 
Table 2. Effect of planting date, cultivar and potassium silicate rate on dry weight of garlic plant at 105 and 135 days after planting during 2016/2017 and 2017/2018 seasons

\begin{tabular}{|c|c|c|c|c|c|c|c|c|c|c|c|c|}
\hline \multirow[t]{4}{*}{ Treatment } & \multicolumn{4}{|c|}{$\begin{array}{c}\text { Dry weight } \\
\text { of leaves/plant (g) }\end{array}$} & \multicolumn{4}{|c|}{$\begin{array}{l}\text { Dry weight } \\
\text { of bulb (g) }\end{array}$} & \multicolumn{4}{|c|}{$\begin{array}{c}\text { Total dry } \\
\text { weight/plant (g) }\end{array}$} \\
\hline & \multicolumn{12}{|c|}{ Days after planting } \\
\hline & \multicolumn{2}{|c|}{105} & \multicolumn{2}{|c|}{135} & \multicolumn{2}{|c|}{105} & \multicolumn{2}{|c|}{135} & \multicolumn{2}{|c|}{105} & \multicolumn{2}{|c|}{135} \\
\hline & S1 & $\mathbf{S 2}$ & S1 & $\mathbf{S 2}$ & S1 & $\mathbf{S 2}$ & S1 & $\mathbf{S 2}$ & S1 & $\mathbf{S 2}$ & S1 & $\mathbf{S 2}$ \\
\hline Planting date & \multicolumn{12}{|c|}{ Effect of planting date } \\
\hline $15^{\text {th }}$ Sep. & 2.82 & 2.81 & 4.21 & 3.40 & 1.38 & 1.19 & 2.42 & 2.35 & 4.20 & 4.00 & 6.63 & 5.75 \\
\hline $1^{\text {st }}$ Oct. & 3.44 & 3.54 & 4.95 & 5.03 & 1.62 & 1.71 & 2.94 & 3.00 & 5.06 & 5.25 & 7.89 & 8.03 \\
\hline $15^{\text {th }}$ Oct. & 3.05 & 3.14 & 4.15 & 3.79 & 1.44 & 1.32 & 2.58 & 2.50 & 4.49 & 4.46 & 6.73 & 6.29 \\
\hline \multirow[t]{2}{*}{ LSD at 0.05 level } & 0.20 & $\mathbf{0 . 0 7}$ & 0.05 & 0.16 & 0.04 & 0.09 & 0.09 & 0.10 & 0.19 & 0.09 & 0.08 & 0.22 \\
\hline & \multicolumn{12}{|c|}{ Effect of cultivar } \\
\hline Balady & 3.24 & 3.26 & 4.54 & 4.24 & 1.59 & 1.51 & 2.74 & 2.73 & 4.83 & 4.77 & 7.28 & 6.97 \\
\hline Sids 40 & 2.97 & 3.06 & 4.33 & 3.91 & 1.37 & 1.30 & 2.55 & 2.50 & 4.34 & 4.36 & 6.88 & 6.41 \\
\hline \multirow[t]{2}{*}{ LSD at 0.05 level } & 0.10 & 0.12 & 0.05 & 0.11 & $\mathbf{0 . 0 3}$ & 0.03 & 0.02 & 0.11 & 0.11 & 0.12 & 0.06 & 0.15 \\
\hline & \multicolumn{12}{|c|}{ Effect of potassium silicate rate } \\
\hline $\mathbf{0}$ & 2.92 & 2.90 & 4.19 & 3.71 & 1.40 & 1.31 & 2.52 & 2.44 & 4.32 & 4.21 & 6.71 & 6.15 \\
\hline $1 \mathrm{ml} / \mathbf{l}$ & 3.15 & 3.13 & 4.44 & 4.05 & 1.48 & 1.37 & 2.63 & 2.66 & 4.63 & 4.50 & 7.07 & 6.71 \\
\hline $2 \mathrm{ml} / \mathrm{l}$ & 3.25 & 3.46 & 4.68 & 4.46 & 1.56 & 1.54 & 2.79 & 2.75 & 4.81 & 5.00 & 7.47 & 7.21 \\
\hline LSD at 0.05 level & 0.10 & 0.10 & 0.08 & 0.11 & 0.04 & 0.04 & 0.03 & 0.12 & 0.10 & 0.10 & 0.09 & 0.19 \\
\hline
\end{tabular}

S1: $1^{\text {st }}$ season and $\mathrm{S} 2: 2^{\text {nd }}$ season

Planting dates plays an important role on garlic growth. Garlic is known to be thermo and photo-sensitive crop (Jones and Mann, 1963) and its vegetative growth and bulb formation are greatly influenced by growing environment (Rahim and Fordham, 1988). These results agree with those reported by Abdalla et al. (2011), Gunda (2013), Vidya et al. (2013), Hassan et al. (2016) and Mohammad et al. (2018) on garlic.

\section{Effect of cultivars}

Balady cultivar gave higher values of dry weight of leaves, bulb and total dry weight/plant than Sids 40 at 105 and 135 DAP in both seasons (Table 2). The differences between garlic cultivars could be attributed to the genetic differences between cultivars. Differences between garlic cultivars were also observed by Youssef and Tony (2014), Hassan (2015), Hassan et al. (2016) and Merwad (2018).

\section{Effect of K silicate}

Dry weight of leaves, bulb and total dry weight/plant significantly increased with increasing $\mathrm{K}$ silicate up to $2 \mathrm{ml} / \mathrm{l}$ at 105 and $135 \mathrm{DAP}$ in both seasons (Table 2). This means that $\mathrm{K}$ silicate at $2 \mathrm{ml} / 1$ increased dry weight of leaves, bulb and total dry weight/plant compared to 1 $\mathrm{ml} / 1$ and control.

The previous positive action of potassium silicate on growth characters because it contains higher amounts of silicon (25\%) might be attributed to its important roles in protecting plants against drought, cold, diseases and fungal attack, alleviating abiotic stress of heavy metals 
toxicity and salinity and improving root development, uptake of water and nutrients and plant pigments (Qin and Tian 2009). The obtained results are in a good accordance with those recorded by Salim et al. (2014) on potato and Abou El-Khair and Mohsen (2016) on Jerusalem artichoke.

\section{Effect of the interaction between planting date and cultivars}

The interaction between planting date and cultivars reflect significant effect on dry weight of leaves/plant, bulb and total dry weight/plant at 105 and 135 DAP in both seasons (Table 3). At 135 DAP, planting Balady cultivar on $1^{\text {st }}$ Oct. gave the highest value for each of dry weight of leaves/plant, bulb and total dry weight/plant in both seasons, followed by planting Sids 40 on $1^{\text {st }}$ October.

\section{Effect of the interaction between planting date and $\mathrm{K}$ silicate}

The interaction between planting date and $\mathrm{K}$ silicate had significant effect on dry weight of leaves/ plant, bulb and total dry weight / plant at 105 and 135 DAP in both seasons (Table 4). At 135 DAP, planting on $1^{\text {st }}$ Oct. and spraying with $\mathrm{K}$ silicate at $2 \mathrm{ml} / 1$ increased dry weight of leaves/plant, bulb and total dry weight/plant, followed by planting on $1^{\text {st }}$ Oct. and spraying with $\mathrm{K}$ silicate at $1 \mathrm{ml} / \mathrm{l}$ in both seasons.

\section{Effect of the interaction between cultivars and $\mathrm{K}$ silicate}

Results in Table 5 indicate that, the interaction between cultivars and $\mathrm{K}$ silicate reflect significant effect on dry weight of leaves/plant, bulb and total dry weight/plant at 105 and 135 DAP. At 135 DAP, spraying Balady cultivar with $\mathrm{K}$ silicate at $2 \mathrm{ml} / \mathrm{l}$ increased dry weight of leaves/ plant, bulb and total dry weight/plant, followed by spraying Balady cultivar with $\mathrm{K}$ silicate at $1 \mathrm{ml} / 1$.

Effect of the triple interaction among planting date, cultivars and $K$ silicate

The interaction among planting date, cultivars and $\mathrm{K}$ silicate had significant effect on dry weight of leaves/plant, bulb and total dry weight/plant at 105 and 135 DAP in both seasons (Table 6). At 135 DAP, planting Balady cultivar on $1^{\text {st }}$ Oct. and spraying with $\mathrm{K}$ silicate at $2 \mathrm{ml} / 1$ and planting Sids 40 cultivar on $1^{\text {st }}$ Oct. and spraying with $\mathrm{K}$ silicate at $2 \mathrm{ml} / 1$ increased dry weight of leaves/ plant, bulb and total dry weight/plant in both seasons.

\section{Yield and its Components}

\section{Effect of planting date}

Planting garlic on $1^{\text {st }}$ Oct., increased yield of grades 1, 2, 3 and 4, exportable, marketable and total yield as well as average bulb weight, followed by planting on $15^{\text {th }}$ Oct. in both seasons (Table 7). The differences between planting dates in total yield/fad., as a result of their variation in the total dry weight (Table 1).

The enhancement of bulb yield and its components as a results of early planting might be due to that the plants received enough earlier cool weather period and a shorter day length which enhanced the vegetative growth of plant before the formation of their bulbs, consequently, resulting in assimilation of more carbohydrates and their translocation to the bulbs, leading to increase head bulb yield and its components (Hassan et al., 2016). Results are in harmony with those reported by Muhammad et al. (2001), Bhuiya et al. (2003), Rahim et al. (2003), Vidya et al. (2013), Youssef and Tony (2014), Choudhary (2015), Hassan et al. (2016) and Mohammad (2018) on garlic.

\section{Effect of cultivars}

Balady cultivar gave higher yield of grades 1 , 2, 3 and 4, exportable, marketable and total yield as well as average bulb weight than Sids 40 cultivar in both seasons (Table 8). These results might be referred to the genetic variation among garlic cultivars and their abilities to benefit from the environmental sources, particularly, light, $\mathrm{CO}_{2}$, water and nutrients.

These results are in agreement with those reported by Abdalla et al. (2011), Mohsen (2012), Abdel-Razzak and El-Sharkawy (2013), Abo El-Fadel and Mohamed (2013) and Merwad (2018). They found that the Balady garlic cultivar gave higher total yield and its components of bulbs, than Sids 40 cultivar.

\section{Effect of K silicate}

Yields of grades 1, 2, 3 and 4, exportable, marketable and total yield as well as average bulb weight significantly increased with increasing $\mathrm{K}$ silicate level at $2 \mathrm{ml} / \mathrm{l}$ in both seasons (Table 9). Spraying garlic plants with $\mathrm{K}$ silicate at $2 \mathrm{ml} / \mathrm{l}$ 
Table 3. Effect of the interaction between planting date and cultivar on dry weight of garlic plant at 105 and 135 days after planting during 2016/2017 and 2017/2018 seasons

\begin{tabular}{|c|c|c|c|c|c|c|c|}
\hline \multirow[t]{3}{*}{ Treatment } & & \multicolumn{2}{|c|}{$\begin{array}{r}\text { Dry weight of } \\
\text { leaves/plant (g) }\end{array}$} & \multicolumn{2}{|c|}{$\begin{array}{l}\text { Dry weight of } \\
\text { bulb (g) }\end{array}$} & \multicolumn{2}{|c|}{$\begin{array}{c}\text { Total dry } \\
\text { weight/plant (g) }\end{array}$} \\
\hline & & \multicolumn{6}{|c|}{ Days after planting } \\
\hline & & 105 & 135 & 105 & 135 & 105 & 135 \\
\hline$\overline{\text { Planting date }}$ & Cultivar & \multicolumn{6}{|c|}{$2016 / 2017$ season } \\
\hline \multirow[t]{2}{*}{$15^{\text {th }}$ Sep. } & Balady & 2.89 & 4.33 & 1.49 & 2.51 & 4.38 & 6.84 \\
\hline & Sids 40 & 2.76 & 4.09 & 1.27 & 2.33 & 4.03 & 6.42 \\
\hline \multirow[t]{2}{*}{$1^{\text {st }}$ Oct. } & Balady & 3.63 & 5.05 & 1.74 & 3.03 & 5.37 & 8.08 \\
\hline & Sids 40 & 3.25 & 4.85 & 1.50 & 2.85 & 4.75 & 7.70 \\
\hline \multirow[t]{2}{*}{$15^{\text {th }}$ Oct. } & Balady & 3.19 & 4.25 & 1.54 & 2.68 & 4.73 & 6.93 \\
\hline & Sids 40 & 2.92 & 4.05 & 1.34 & 2.48 & 4.26 & 6.53 \\
\hline \multicolumn{2}{|c|}{ LSD at 0.05 level } & 0.17 & 0.10 & 0.05 & 0.03 & 0.19 & 0.11 \\
\hline & & \multicolumn{6}{|c|}{$2017 / 2018$ season } \\
\hline \multirow{2}{*}{$15^{\text {th }}$ Sep. } & Balady & 2.86 & 3.56 & 1.27 & 2.50 & 4.13 & 6.06 \\
\hline & Sids 40 & 2.77 & 3.24 & 1.10 & 2.20 & 3.87 & 5.44 \\
\hline \multirow[t]{2}{*}{$1^{\text {st }}$ Oct. } & Balady & 3.70 & 5.13 & 1.84 & 3.10 & 5.54 & 8.23 \\
\hline & Sids 40 & 3.39 & 4.93 & 1.58 & 2.90 & 4.97 & 7.83 \\
\hline \multirow[t]{2}{*}{$15^{\text {th }}$ Oct. } & Balady & 3.24 & 4.02 & 1.40 & 2.60 & 4.64 & 6.62 \\
\hline & Sids 40 & 3.04 & 3.56 & 1.24 & 2.40 & 4.28 & 5.96 \\
\hline \multicolumn{2}{|c|}{ LSD at 0.05 level } & 0.21 & 0.19 & 0.05 & 0.19 & 0.21 & 0.26 \\
\hline
\end{tabular}

Table 4. Effect of the interaction between planting date and potassium silicate rate on dry weight of garlic plant at 105 and 135 days after planting during 2016/2017 and 2017/ 2018 seasons

\begin{tabular}{|c|c|c|c|c|c|c|c|}
\hline \multirow[t]{3}{*}{ Treatment } & & \multicolumn{2}{|c|}{$\begin{array}{l}\text { Dry weight of } \\
\text { leaves/plant (g) }\end{array}$} & \multicolumn{2}{|c|}{$\begin{array}{l}\text { Dry weight of } \\
\text { bulb (g) }\end{array}$} & \multicolumn{2}{|c|}{$\begin{array}{c}\text { Total dry } \\
\text { weight/plant (g) }\end{array}$} \\
\hline & & \multicolumn{6}{|c|}{ Days after planting } \\
\hline & & 105 & 135 & 105 & 135 & 105 & 135 \\
\hline Planting date & $\mathrm{K}$ silicate rate & \multicolumn{6}{|c|}{ 2016/2017 season } \\
\hline \multirow{3}{*}{$15^{\text {th }}$ Sep. } & $\mathbf{0}$ & 2.66 & 4.12 & 1.33 & 2.25 & 3.99 & 6.37 \\
\hline & $1 \mathrm{ml} / \mathrm{l}$ & 2.83 & 4.17 & 1.37 & 2.38 & 4.2 & 6.55 \\
\hline & $2 \mathrm{ml} / \mathrm{l}$ & 2.98 & 4.34 & 1.45 & 2.64 & 4.43 & 6.98 \\
\hline \multirow{3}{*}{$1^{\text {st }}$ Oct. } & $\mathbf{0}$ & 3.30 & 4.75 & 1.52 & 2.85 & 4.82 & 7.6 \\
\hline & $1 \mathrm{ml} / \mathrm{l}$ & 3.48 & 4.94 & 1.63 & 2.91 & 5.11 & 7.85 \\
\hline & $2 \mathrm{ml} / \mathrm{l}$ & 3.54 & 5.17 & 1.72 & 3.06 & 5.26 & 8.23 \\
\hline \multirow[t]{3}{*}{$15^{\text {th }}$ Oct. } & 0 & 2.79 & 3.70 & 1.36 & 2.48 & 4.15 & 6.18 \\
\hline & $1 \mathrm{ml} / \mathbf{l}$ & 3.14 & 4.22 & 1.44 & 2.60 & 4.58 & 6.82 \\
\hline & $2 \mathrm{ml} / \mathrm{l}$ & 3.23 & 4.54 & 1.52 & 2.25 & 4.75 & 6.79 \\
\hline \multicolumn{2}{|c|}{ LSD at 0.05 level } & 0.18 & 0.14 & 0.07 & 0.05 & 0.10 & 0.15 \\
\hline & & \multicolumn{6}{|c|}{ 2017/2018 season } \\
\hline \multirow{3}{*}{$15^{\text {th }}$ Sep. } & $\mathbf{0}$ & 2.38 & 2.98 & 1.10 & 2.14 & 3.48 & 5.12 \\
\hline & $1 \mathrm{ml} / \mathrm{l}$ & 2.77 & 3.17 & 1.16 & 2.47 & 3.93 & 5.64 \\
\hline & $2 \mathrm{ml} / \mathrm{l}$ & 3.30 & 4.06 & 1.30 & 2.44 & 4.6 & 6.5 \\
\hline \multirow[t]{3}{*}{$1^{\text {st }}$ Oct. } & 0 & 3.36 & 4.71 & 1.57 & 2.84 & 4.93 & 7.55 \\
\hline & $1 \mathrm{ml} / \mathrm{l}$ & 3.52 & 5.06 & 1.66 & 3.01 & 5.18 & 8.07 \\
\hline & $2 \mathrm{ml} / \mathrm{l}$ & 3.75 & 5.33 & 1.90 & 3.14 & 5.65 & 8.47 \\
\hline \multirow{3}{*}{$15^{\text {th }}$ Oct. } & 0 & 2.97 & 3.45 & 1.26 & 2.34 & 4.23 & 5.79 \\
\hline & $1 \mathrm{ml} / \mathbf{l}$ & 3.10 & 3.93 & 1.29 & 2.50 & 4.39 & 6.43 \\
\hline & $2 \mathrm{ml} / \mathrm{l}$ & 3.34 & 3.99 & 1.42 & 2.67 & 4.76 & 6.66 \\
\hline \multicolumn{2}{|c|}{ LSD at 0.05 level } & 0.18 & 0.20 & 0.07 & 0.21 & 0.17 & 0.34 \\
\hline
\end{tabular}


Table 5. Effect of the interaction between cultivar and potassium silicate rate on dry weight of garlic plant at 105 and 135 days after planting during 2016/2017 and 2017/2018 seasons

\begin{tabular}{|c|c|c|c|c|c|c|c|}
\hline \multirow[t]{3}{*}{ Treatment } & & \multicolumn{2}{|c|}{$\begin{array}{l}\text { Dry weight of } \\
\text { leaves/plant (g) }\end{array}$} & \multicolumn{2}{|c|}{$\begin{array}{c}\text { Dry weight of } \\
\text { bulb (g) }\end{array}$} & \multicolumn{2}{|c|}{$\begin{array}{c}\text { Total dry } \\
\text { weight/plant (g) }\end{array}$} \\
\hline & & \multicolumn{6}{|c|}{ Days after planting } \\
\hline & & 105 & 135 & 105 & 135 & 105 & 135 \\
\hline$\overline{\text { Cultivar }}$ & K silicate rate & & & 2016 & $\overline{\text { season }}$ & & \\
\hline \multirow{3}{*}{ Balady } & $\mathbf{0}$ & 3.05 & 4.33 & 1.52 & 2.62 & 4.57 & 6.95 \\
\hline & $1 \mathrm{ml} / \mathbf{l}$ & 3.26 & 4.52 & 1.59 & 2.72 & 4.85 & 7.24 \\
\hline & $2 \mathrm{ml} / \mathrm{l}$ & 3.41 & 4.78 & 1.66 & 2.88 & 5.07 & 7.66 \\
\hline \multirow[t]{3}{*}{ Sids 40} & $\mathbf{0}$ & 2.78 & 4.05 & 1.28 & 2.43 & 4.06 & 6.48 \\
\hline & $1 \mathrm{ml} / \mathbf{l}$ & 3.04 & 4.36 & 1.37 & 2.53 & 4.41 & 6.89 \\
\hline & $2 \mathrm{ml} / \mathrm{l}$ & 3.10 & 4.58 & 1.46 & 2.69 & 4.56 & 7.27 \\
\hline \multirow{2}{*}{\multicolumn{2}{|c|}{ LSD at 0.05 level }} & 0.15 & 0.12 & 0.05 & 0.04 & 0.15 & 0.12 \\
\hline & & \multicolumn{6}{|c|}{$2017 / 2018$ season } \\
\hline \multirow[t]{3}{*}{ Balady } & $\mathbf{0}$ & 3.06 & 3.88 & 1.41 & 2.54 & 4.47 & 6.42 \\
\hline & $1 \mathrm{ml} / \mathbf{l}$ & 3.23 & 4.17 & 1.46 & 2.85 & 4.69 & 7.02 \\
\hline & $2 \mathrm{ml} / \mathrm{l}$ & 3.51 & 4.66 & 1.65 & 2.81 & 5.16 & 7.47 \\
\hline \multirow[t]{3}{*}{ Sids 40} & $\mathbf{0}$ & 2.74 & 3.54 & 1.21 & 2.34 & 3.95 & 5.88 \\
\hline & $1 \mathrm{ml} / \mathbf{l}$ & 3.03 & 3.93 & 1.28 & 2.47 & 4.31 & 6.4 \\
\hline & $2 \mathrm{ml} / \mathrm{l}$ & 3.42 & 4.25 & 1.43 & 2.69 & 4.85 & 6.94 \\
\hline \multicolumn{2}{|c|}{ LSD at 0.05 level } & 0.15 & 0.16 & 0.06 & 0.17 & 0.14 & 0.27 \\
\hline
\end{tabular}

Table 6. Effect of the triple interaction among planting date, cultivar and $\mathrm{K}$ silicate rate on dry weight of garlic plant at 105 and 135 days after planting during 2016/2017 and 2017/ 2018 seasons

\begin{tabular}{|c|c|c|c|c|c|c|c|c|c|c|c|c|c|c|}
\hline \multirow{3}{*}{$\begin{array}{l}\text { Treatment } \\
\text { Planting } \\
\text { date }\end{array}$} & \multirow{3}{*}{ Cultivar } & \multirow{3}{*}{$\begin{array}{l}\text { K silicate } \\
\text { rate }\end{array}$} & \multicolumn{4}{|c|}{$\begin{array}{l}\text { Dry weight of } \\
\text { leaves/plant (g) }\end{array}$} & \multicolumn{4}{|c|}{$\begin{array}{l}\text { Dry weight of } \\
\text { bulb (g) }\end{array}$} & \multicolumn{4}{|c|}{$\begin{array}{c}\text { Total dry } \\
\text { weight/plant (g) }\end{array}$} \\
\hline & & & \multicolumn{12}{|c|}{ Days after planting } \\
\hline & & & \multicolumn{2}{|c|}{105} & \multicolumn{2}{|c|}{135} & \multicolumn{2}{|c|}{105} & \multicolumn{2}{|c|}{135} & \multicolumn{2}{|c|}{105} & \multicolumn{2}{|c|}{135} \\
\hline & & & S1 & S2 & S1 & $\overline{\text { S2 }}$ & $\overline{S 1}$ & $\mathbf{S 2}$ & S1 & S2 & $\overline{\text { S1 }}$ & $\mathbf{S 2}$ & $\overline{\text { S1 }}$ & $\mathbf{S 2}$ \\
\hline \multirow[t]{6}{*}{$15^{\text {th }}$ Sep. } & Balady & $\mathbf{0}$ & 2.74 & 2.48 & 4.34 & 3.30 & 1.43 & 1.20 & 2.35 & 2.25 & 4.17 & 3.68 & 6.69 & 5.55 \\
\hline & & $1 \mathrm{ml} / \mathbf{l}$ & 2.92 & 2.88 & 4.21 & 3.27 & 1.50 & 1.26 & 2.48 & 2.84 & 4.42 & 4.14 & 6.69 & 6.11 \\
\hline & & $2 \mathrm{ml} / \mathrm{l}$ & 3.03 & 3.23 & 4.44 & 4.12 & 1.55 & 1.36 & 2.71 & 2.43 & 4.58 & 4.59 & 7.15 & 6.55 \\
\hline & Sids 40 & $\mathbf{0}$ & 2.58 & 2.28 & 3.90 & 2.66 & 1.22 & 1.00 & 2.15 & 2.04 & 3.80 & 3.28 & 6.05 & 4.70 \\
\hline & & $1 \mathrm{ml} / \mathbf{l}$ & 2.75 & 2.66 & 4.13 & 3.07 & 1.24 & 1.06 & 2.28 & 2.10 & 3.99 & 3.72 & 6.41 & 5.17 \\
\hline & & $2 \mathrm{ml} / \mathrm{l}$ & 2.94 & 3.36 & 4.24 & 3.99 & 1.35 & 1.25 & 2.56 & 2.45 & 4.29 & 4.61 & 6.80 & 6.44 \\
\hline \multirow[t]{6}{*}{$1^{\text {st }}$ Oct. } & Balady & 0 & 3.50 & 3.62 & 4.85 & 4.81 & 1.67 & 1.67 & 2.93 & 2.94 & 5.17 & 5.29 & 7.78 & 7.75 \\
\hline & & $1 \mathrm{ml} / \mathbf{l}$ & 3.64 & 3.62 & 5.04 & 5.16 & 1.74 & 1.76 & 3.00 & 3.11 & 5.38 & 5.38 & 8.04 & 8.27 \\
\hline & & $2 \mathrm{ml} / \mathrm{l}$ & 3.75 & 3.85 & 5.27 & 5.43 & 1.82 & 2.10 & 3.17 & 3.24 & 5.57 & 5.95 & 8.44 & 8.67 \\
\hline & Sids 40 & $\mathbf{0}$ & 3.09 & 3.09 & 4.65 & 4.61 & 1.36 & 1.47 & 2.76 & 2.74 & 4.45 & 4.56 & 7.41 & 7.35 \\
\hline & & $1 \mathrm{ml} / \mathrm{l}$ & 3.32 & 3.42 & 4.84 & 4.96 & 1.53 & 1.56 & 2.83 & 2.91 & 4.85 & 4.98 & 7.67 & 7.87 \\
\hline & & $2 \mathrm{ml} / \mathrm{l}$ & 3.34 & 3.65 & 5.07 & 5.23 & 1.62 & 1.70 & 2.96 & 3.04 & 4.96 & 5.35 & 8.03 & 8.27 \\
\hline \multirow[t]{6}{*}{$15^{\text {th }}$ Oct. } & Balady & 0 & 2.90 & 3.07 & 3.80 & 3.53 & 1.46 & 1.36 & 2.58 & 2.44 & 4.36 & 4.43 & 6.38 & 5.97 \\
\hline & & $1 \mathrm{ml} / \mathrm{l}$ & 3.24 & 3.20 & 4.32 & 4.10 & 1.54 & 1.35 & 2.70 & 2.60 & 4.78 & 4.55 & 7.02 & 6.70 \\
\hline & & $2 \mathrm{ml} / \mathrm{l}$ & 3.44 & 3.44 & 4.64 & 4.44 & 1.62 & 1.51 & 2.77 & 2.77 & 5.06 & 4.95 & 7.41 & 7.21 \\
\hline & Sids 40 & $\mathbf{0}$ & 2.68 & 2.87 & 3.60 & 3.36 & 1.26 & 1.16 & 2.38 & 2.24 & 3.94 & 4.03 & 5.98 & 5.60 \\
\hline & & $1 \mathrm{ml} / \mathrm{l}$ & 3.05 & 3.00 & 4.12 & 3.76 & 1.34 & 1.22 & 2.50 & 2.40 & 4.39 & 4.22 & 6.62 & 6.16 \\
\hline & & $2 \mathrm{ml} / \mathrm{l}$ & 3.02 & 3.24 & 4.44 & 3.54 & 1.42 & 1.33 & 2.56 & 2.57 & 4.44 & 4.57 & 7.00 & 6.11 \\
\hline \multicolumn{2}{|c|}{ LSD at 0.05 level } & & 0.26 & 0.26 & 0.20 & 0.28 & 0.09 & 0.10 & 0.07 & 0.31 & 0.26 & 0.25 & 0.22 & 0.48 \\
\hline
\end{tabular}


Table 7. Effect of planting date on yield and its components of garlic during 2016/2017 and $2017 / 2018$ seasons

\begin{tabular}{|c|c|c|c|c|c|c|c|c|}
\hline \multirow[t]{2}{*}{ Treatment } & \multicolumn{7}{|c|}{ Yield and its components (ton/fad.) } & \multirow{2}{*}{$\begin{array}{c}\text { Average } \\
\text { bulb weigh } \\
\text { (g) }\end{array}$} \\
\hline & $\begin{array}{c}\text { Grade } \\
\text { (1) }\end{array}$ & $\begin{array}{c}\text { Grade } \\
\text { (2) }\end{array}$ & $\begin{array}{c}\text { Grade } \\
\text { (3) }\end{array}$ & $\begin{array}{c}\text { Grade } \\
(4)\end{array}$ & $\begin{array}{c}\text { Exportable } \\
\text { yield } \\
\end{array}$ & $\begin{array}{l}\text { Marketable } \\
\text { yield }\end{array}$ & $\begin{array}{l}\text { Total } \\
\text { yield }\end{array}$ & \\
\hline Planting date & \multicolumn{8}{|c|}{$2016 / 2017$ season } \\
\hline $15^{\text {th }}$ Sep. & 1.998 & 1.668 & 1.343 & 0.820 & 3.666 & 5.009 & 5.829 & 43.08 \\
\hline $1^{\text {st }}$ Oct. & 2.311 & 2.183 & 1.694 & 1.140 & 4.494 & 6.188 & 7.328 & 52.07 \\
\hline $15^{\text {th }}$ Oct. & 2.160 & 1.775 & 1.520 & 0.899 & 3.935 & 5.455 & 6.354 & 46.40 \\
\hline \multirow[t]{2}{*}{ LSD at 0.05 level } & 0.050 & 0.071 & 0.049 & $\mathbf{0 . 0 3 7}$ & 0.054 & 0.042 & 0.056 & 0.68 \\
\hline & \multicolumn{8}{|c|}{$2017 / 2018$ season } \\
\hline $15^{\text {th }}$ Sep. & 1.980 & 1.147 & 1.301 & 0.815 & 3.127 & 4.428 & 5.243 & 40.62 \\
\hline $1^{\text {st }}$ Oct. & 2.323 & 1.494 & 1.687 & 1.144 & 3.817 & 5.504 & 6.648 & 50.60 \\
\hline $15^{\text {th }}$ Oct. & 2.158 & 1.283 & 1.527 & 0.945 & 3.441 & 4.968 & 5.913 & 40.87 \\
\hline LSD at 0.05 level & 0.047 & 0.283 & 0.040 & 0.070 & 0.303 & 0.323 & 0.310 & 3.77 \\
\hline
\end{tabular}

Table 8. Effect of cultivar on yield and its components of garlic during 2016/2017 and 2017/2018 seasons

\begin{tabular}{|c|c|c|c|c|c|c|c|c|}
\hline \multirow[t]{2}{*}{ Treatment } & \multicolumn{7}{|c|}{ Yield and its components (ton/fad.) } & \multirow{2}{*}{$\begin{array}{l}\text { Average } \\
\text { bulb weight } \\
\text { (g) }\end{array}$} \\
\hline & $\begin{array}{c}\text { Grade } \\
\text { (1) }\end{array}$ & $\begin{array}{c}\text { Grade } \\
(2)\end{array}$ & $\begin{array}{c}\text { Grade } \\
\text { (3) }\end{array}$ & $\begin{array}{c}\text { Grade } \\
\text { (4) }\end{array}$ & $\begin{array}{c}\text { Exportable } \\
\text { yield } \\
\end{array}$ & $\begin{array}{c}\text { Marketable } \\
\text { yield }\end{array}$ & $\begin{array}{l}\text { Total } \\
\text { yield } \\
\end{array}$ & \\
\hline Cultivar & \multicolumn{8}{|c|}{$2016 / 2017$ season } \\
\hline Balady & 2.295 & 2.011 & 1.659 & 1.090 & 4.306 & 5.965 & 7.055 & 47.43 \\
\hline Sids 40 & 2.018 & 1.739 & 1.379 & 0.817 & 3.757 & 5.136 & 5.953 & 46.94 \\
\hline \multirow[t]{2}{*}{ LSD at 0.05 level } & 0.097 & $\mathbf{0 . 0 3 0}$ & 0.012 & 0.030 & 0.098 & 0.022 & 0.020 & 0.20 \\
\hline & \multicolumn{8}{|c|}{$2017 / 2018$ season } \\
\hline Balady & 2.291 & 1.49 & 1.643 & 1.106 & 3.781 & 5.424 & 6.530 & 44.20 \\
\hline Sids 40 & 2.016 & 1.126 & 1.368 & 0.829 & 3.142 & 4.51 & 5.339 & 43.19 \\
\hline LSD at 0.05 level & 0.032 & 0.160 & 0.077 & 0.034 & 0.160 & 0.156 & 0.155 & 0.56 \\
\hline
\end{tabular}


Table 9. Effect of potassium silicate rate on yield and its components of garlic during 2016/2017 and 2017/2018 seasons

\begin{tabular}{|c|c|c|c|c|c|c|c|c|}
\hline \multirow[t]{2}{*}{ Treatment } & \multicolumn{7}{|c|}{ Yield and its components (ton/fad.) } & \multirow{2}{*}{$\begin{array}{l}\text { Average } \\
\text { bulb weight } \\
\text { (g) }\end{array}$} \\
\hline & $\begin{array}{c}\text { Grade } \\
\text { (1) }\end{array}$ & $\begin{array}{c}\text { Grade } \\
\text { (2) }\end{array}$ & $\begin{array}{c}\text { Grade } \\
\text { (3) }\end{array}$ & $\begin{array}{c}\text { Grade } \\
(4)\end{array}$ & $\begin{array}{c}\text { Exportable } \\
\text { yield } \\
\end{array}$ & $\begin{array}{c}\text { Marketable } \\
\text { yield }\end{array}$ & $\begin{array}{l}\text { Total } \\
\text { yield }\end{array}$ & \\
\hline K silicate rate & \multicolumn{8}{|c|}{$2016 / 2017$ season } \\
\hline $\mathbf{0}$ & 2.035 & 1.752 & 1.393 & 0.854 & 3.787 & 5.180 & 6.034 & 44.46 \\
\hline $1 \mathrm{ml} / \mathbf{l}$ & 2.153 & 1.897 & 1.532 & 0.956 & 4.050 & 5.582 & 6.538 & 47.57 \\
\hline $2 \mathrm{ml} / \mathrm{l}$ & 2.282 & 1.977 & 1.631 & 1.050 & 4.259 & 5.890 & 6.940 & 49.51 \\
\hline \multirow[t]{2}{*}{ LSD at 0.05 level } & 0.016 & 0.020 & 0.031 & 0.020 & 0.021 & 0.034 & 0.037 & 0.37 \\
\hline & \multicolumn{8}{|c|}{$2017 / 2018$ season } \\
\hline $\mathbf{0}$ & 2.024 & 1.239 & 1.391 & 0.852 & 3.263 & 4.654 & 5.506 & 40.44 \\
\hline $1 \mathrm{ml} / \mathbf{l}$ & 2.153 & 1.31 & 1.516 & 0.955 & 3.463 & 4.979 & 5.934 & 42.91 \\
\hline $2 \mathrm{ml} / \mathrm{l}$ & 2.284 & 1.375 & 1.609 & 1.096 & 3.659 & 5.268 & 6.364 & 47.74 \\
\hline LSD at 0.05 level & 0.030 & 0.129 & 0.026 & 0.048 & 0.127 & 0.131 & 0.141 & 2.85 \\
\hline
\end{tabular}

increased yield of grades $1,2,3$ and 4 , exportable, marketable and total yield as well as average bulb weight in both seasons. Results are in harmony with those reported by Wand and Galletta (1998) on strawberries, Abou-Baker et al. (2011) on bean, Kamal (2013) on sweet pepper, (Salim et al., 2014) on potato, Abou ElKhair and Mohsen (2016) on Jerusalem artichoke and Elrys and Merwad (2017) on pea.

\section{Effect of the interaction between planting date and cultivars}

The interaction between planting date and cultivars had significant effect on yield and its components in both seasons (Table 10). Planting Balady cultivar on $1^{\text {st }}$ Oct. increased yield of grades 1,2,3 and 4, exportable, marketable and total yields as well as average bulb weight in both seasons, followed by planting Balady cultivar on $15^{\text {th }}$ Oct. and planting Sids 40 cultivar on $1^{\text {st }}$ Oct. in both seasons.

\section{Effect of the interaction between planting date and $K$ silicate}

The interaction between planting date and $\mathrm{K}$ silicate reflect significant effect on yields and its components in both seasons (Table 11). Planting on $1^{\text {st }}$ Oct. and spraying with $\mathrm{K}$ silicate at $2 \mathrm{ml} / \mathrm{l}$ increased yield of grades 1, 2, 3 and 4, exportable, marketable and total yields as well as average bulb weight, followed by planting on $1^{\text {st }}$ Oct. and spraying with $\mathrm{K}$ silicate at $1 \mathrm{ml} / 1$ in both seasons.

\section{Effect of the interaction between cultivars and $K$ silicate}

Results in Table 12 show that, the interaction between cultivars and $\mathrm{K}$ silicate reflect significant effect on yield and its components in both seasons. Spraying Balady cultivar with K silicate at $2 \mathrm{ml} / 1$ increased yield of grades 1,2,3 and 4, exportable, marketable and total yields, followed by spraying Balady cultivar with $\mathrm{K}$ silicate at $1 \mathrm{ml} / 1$ in both seasons. As for average bulb weight, spraying Balady and Sids 40 cultivars with $\mathrm{K}$ silicate at $2 \mathrm{ml} / 1$ increased average bulb weight in both seasons.

Effect of the triple interaction among planting date, cultivar and $K$ silicate

The interaction among planting date, cultivar and $\mathrm{K}$ silicate had significant effect on yield and its components in both seasons (Tables 13 and 14). Planting Balady cultivar on $1^{\text {st }}$ Oct. and spraying with $\mathrm{K}$ silicate at $2 \mathrm{ml} / 1$ increased yield of grades 1, 2, 3 and 4, exportable, marketable and total yields as well as average bulb weight with no significant differences with 
Table 10. Effect of the interaction between planting dates and cultivars on yield and its components of garlic during 2016/2017 and 2017/2018 seasons

\begin{tabular}{|c|c|c|c|c|c|c|c|c|c|}
\hline \multirow[t]{2}{*}{ Treatment } & & \multicolumn{7}{|c|}{ Yield and its components (ton/fad.) } & \multirow{2}{*}{$\begin{array}{c}\text { Average } \\
\text { bulb weight } \\
\text { (g) }\end{array}$} \\
\hline & & $\begin{array}{c}\text { Grade } \\
\text { (1) }\end{array}$ & $\begin{array}{c}\text { Grade } \\
\text { (2) }\end{array}$ & $\begin{array}{c}\text { Grade } \\
\text { (3) }\end{array}$ & $\begin{array}{c}\text { Grade } \\
\text { (4) }\end{array}$ & $\begin{array}{c}\text { Exportable } \\
\text { yield }\end{array}$ & $\begin{array}{c}\text { Marketable } \\
\text { yield }\end{array}$ & $\begin{array}{l}\text { Total } \\
\text { yield } \\
\end{array}$ & \\
\hline \multicolumn{2}{|c|}{ Planting date Cultivar } & \multicolumn{8}{|c|}{ 2016/2017 season } \\
\hline \multirow[t]{2}{*}{$15^{\text {th }}$ Sep. } & Balady & 2.134 & 1.805 & 1.482 & 0.957 & 3.939 & 5.421 & 6.378 & 43.39 \\
\hline & Seds 40 & 1.863 & 1.531 & 1.205 & 0.682 & 3.394 & 4.599 & 5.281 & 42.78 \\
\hline \multirow[t]{2}{*}{$1^{\text {st }}$ Oct. } & Balady & 2.449 & 2.317 & 1.831 & 1.275 & 4.766 & 6.597 & 7.872 & 52.26 \\
\hline & Seds 40 & 2.172 & 2.049 & 1.557 & 1.006 & 4.221 & 5.778 & 6.784 & 51.87 \\
\hline \multirow[t]{2}{*}{$15^{\text {th }}$ Oct. } & Balady & 2.302 & 1.911 & 1.664 & 1.037 & 4.213 & 5.877 & 6.914 & 46.63 \\
\hline & Seds 40 & 2.019 & 1.639 & 1.375 & 0.762 & 3.658 & 5.033 & 5.795 & 46.16 \\
\hline \multicolumn{2}{|c|}{ LSD at 0.05 level } & 0.016 & 0.052 & 0.022 & 0.053 & 0.017 & 0.038 & 0.035 & 0.36 \\
\hline & & \multicolumn{8}{|c|}{ 2017/2018 season } \\
\hline \multirow[t]{2}{*}{$15^{\text {th }}$ Sep. } & Balady & 2.117 & 1.272 & 1.439 & 0.955 & 3.389 & 4.828 & 5.783 & 38.55 \\
\hline & Seds 40 & 1.843 & 1.022 & 1.164 & 0.674 & 2.865 & 4.029 & 4.703 & 38.69 \\
\hline \multirow{2}{*}{$1^{\text {st }}$ Oct. } & Balady & 2.462 & 1.777 & 1.822 & 1.280 & 4.239 & 6.061 & 7.341 & 48.58 \\
\hline & Seds 40 & 2.184 & 1.21 & 1.552 & 1.008 & 3.394 & 4.946 & 5.954 & 48.62 \\
\hline \multirow[t]{2}{*}{$15^{\text {th }}$ Oct. } & Balady & 2.295 & 1.421 & 1.667 & 1.084 & 3.716 & 5.383 & 6.467 & 45.48 \\
\hline & Seds 40 & 2.021 & 1.144 & 1.387 & 0.806 & 3.165 & 4.552 & 5.358 & 42.27 \\
\hline \multicolumn{2}{|c|}{ LSD at 0.05 level } & 0.056 & 0.277 & 0.013 & 0.059 & 0.277 & 0.270 & 0.269 & 4.67 \\
\hline
\end{tabular}

Table 11. Effect of the interaction between planting dates and potassium silicate rates on yield and its components of garlic during 2016/2017 and 2017/2018 seasons

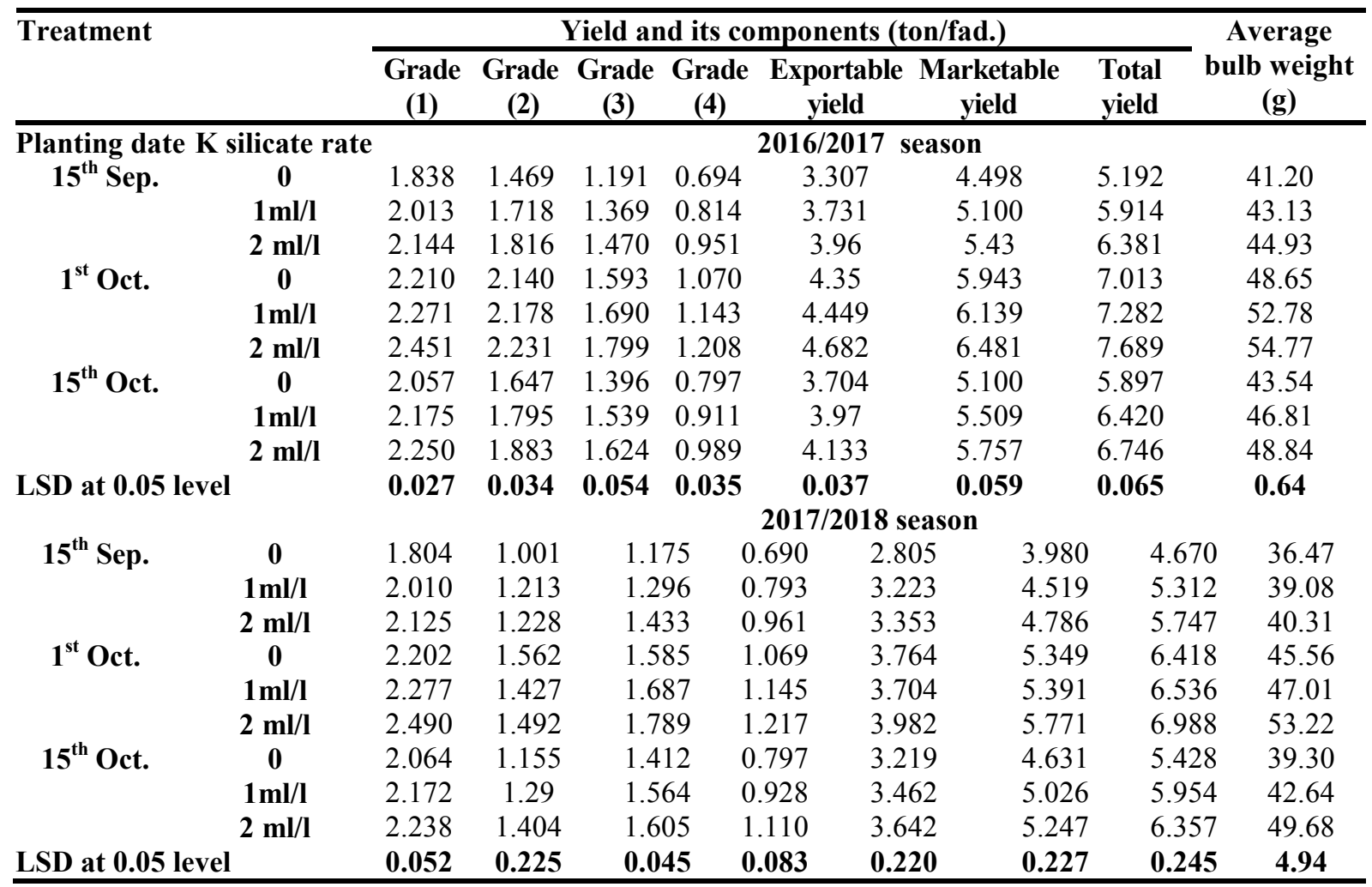


Table 12. Effect of the interaction between cultivars and potassium silicate rates on yield and its components of garlic during 2016/2017 and 2017/2018 seasons

\begin{tabular}{|c|c|c|c|c|c|c|c|c|c|}
\hline \multirow[t]{2}{*}{$\overline{\text { Treatmen }}$} & & \multicolumn{7}{|c|}{ Yield and its components (ton/fad.) } & \multirow{2}{*}{$\begin{array}{c}\text { Average } \\
\text { bulb weight } \\
\text { (g) }\end{array}$} \\
\hline & & $\begin{array}{c}\text { Grade } \\
\text { (1) }\end{array}$ & $\begin{array}{c}\text { Grade } \\
(2)\end{array}$ & $\begin{array}{c}\text { Grade } \\
\text { (3) }\end{array}$ & $\begin{array}{c}\text { Grade } \\
\text { (4) }\end{array}$ & $\begin{array}{c}\text { Exportable } \\
\text { yield }\end{array}$ & $\begin{array}{c}\text { Marketable } \\
\text { yield }\end{array}$ & $\begin{array}{l}\text { Total } \\
\text { yield }\end{array}$ & \\
\hline Cultivar & K silicate rate & \multicolumn{8}{|c|}{$2016 / 2017$ season } \\
\hline \multirow[t]{3}{*}{ Balady } & $\mathbf{0}$ & 2.173 & 1.883 & 1.531 & 0.992 & 4.056 & 5.587 & 6.579 & 44.74 \\
\hline & $1 \mathrm{ml} / \mathbf{l}$ & 2.289 & 2.036 & 1.680 & 1.089 & 4.325 & 6.005 & 7.094 & 47.85 \\
\hline & $2 \mathrm{ml} / \mathrm{l}$ & 2.423 & 2.114 & 1.767 & 1.187 & 4.537 & 6.304 & 7.491 & 49.70 \\
\hline \multirow[t]{3}{*}{ Sids 40} & $\mathbf{0}$ & 1.897 & 1.621 & 1.256 & 0.715 & 3.518 & 4.774 & 5.489 & 44.19 \\
\hline & $1 \mathrm{ml} / \mathbf{l}$ & 2.017 & 1.758 & 1.385 & 0.823 & 3.775 & 5.16 & 5.983 & 47.30 \\
\hline & $2 \mathrm{ml} / \mathrm{l}$ & 2.141 & 1.839 & 1.495 & 0.912 & 3.98 & 5.475 & 6.387 & 49.33 \\
\hline \multirow{2}{*}{\multicolumn{2}{|c|}{ LSD at 0.05 level }} & 0.022 & 0.028 & 0.044 & 0.029 & 0.030 & 0.048 & 0.053 & 0.52 \\
\hline & & \multicolumn{8}{|c|}{$2017 / 2018$ season } \\
\hline \multirow[t]{3}{*}{ Balady } & $\mathbf{0}$ & 2.162 & 1.376 & 1.528 & 0.991 & 3.538 & 5.066 & 6.057 & 40.37 \\
\hline & $1 \mathrm{ml} / \mathbf{l}$ & 2.290 & 1.53 & 1.654 & 1.093 & 3.82 & 5.474 & 6.567 & 42.89 \\
\hline & $2 \mathrm{ml} / \mathrm{l}$ & 2.422 & 1.564 & 1.746 & 1.235 & 3.986 & 5.732 & 6.967 & 49.35 \\
\hline \multirow[t]{3}{*}{ Sids 40} & $\mathbf{0}$ & 1.885 & 1.102 & 1.254 & 0.713 & 2.987 & 4.241 & 4.954 & 40.52 \\
\hline & $1 \mathrm{ml} / \mathbf{l}$ & 2.015 & 1.089 & 1.377 & 0.818 & 3.104 & 4.481 & 5.299 & 42.93 \\
\hline & $2 \mathrm{ml} / \mathrm{l}$ & 2.147 & 1.185 & 1.472 & 0.957 & 3.332 & 4.804 & 5.761 & 46.13 \\
\hline \multicolumn{2}{|c|}{ LSD at 0.05 level } & 0.042 & 0.183 & 0.036 & 0.067 & 0.179 & 0.185 & 0.200 & 4.03 \\
\hline
\end{tabular}

Table 13. Effect of the triple interaction among planting date, cultivar and potassium silicate rate on yield and its components of garlic during 2016/2017 season

\begin{tabular}{|c|c|c|c|c|c|c|c|c|c|c|}
\hline \multirow[t]{2}{*}{ Treatment } & & & \multicolumn{7}{|c|}{ Yield and its components (ton/fad.) } & \multirow{2}{*}{$\begin{array}{c}\text { Average } \\
\text { bulb weight } \\
\text { (g) } \\
\end{array}$} \\
\hline & & & $\begin{array}{l}\text { Grade } \\
\text { (1) }\end{array}$ & $\begin{array}{l}\text { Grade } \\
\text { (2) }\end{array}$ & $\begin{array}{c}\text { Grade } \\
\text { (3) }\end{array}$ & $\begin{array}{c}\text { Grade } \\
\text { (4) }\end{array}$ & $\begin{array}{c}\text { Exportable } \\
\text { yield }\end{array}$ & $\begin{array}{c}\text { Marketable } \\
\text { yield }\end{array}$ & $\begin{array}{l}\text { Total } \\
\text { yield }\end{array}$ & \\
\hline \multicolumn{11}{|c|}{ Planting date Cultivar K silicate rate } \\
\hline \multirow[t]{6}{*}{$15^{\text {th }}$ Sep. } & Balady & 0 & 1.975 & 1.603 & 1.330 & 0.830 & 3.578 & 4.908 & 5.738 & 41.51 \\
\hline & & $1 \mathrm{ml} / \mathrm{l}$ & 2.151 & 1.857 & 1.507 & 0.950 & 4.008 & 5.515 & 6.465 & 43.40 \\
\hline & & $2 \mathrm{ml} / \mathrm{l}$ & 2.275 & 1.956 & 1.609 & 1.080 & 4.231 & 5.840 & 6.920 & 45.26 \\
\hline & Sids 40 & 0 & 1.700 & 1.336 & 1.053 & 0.550 & 3.036 & 4.089 & 4.639 & 40.89 \\
\hline & & $1 \mathrm{ml} / \mathbf{l}$ & 1.875 & 1.579 & 1.230 & 0.670 & 3.454 & 4.684 & 5.354 & 42.86 \\
\hline & & $2 \mathrm{ml} / \mathrm{l}$ & 2.014 & 1.677 & 1.332 & 0.810 & 3.691 & 5.023 & 5.833 & 44.60 \\
\hline \multirow{6}{*}{$1^{\text {st }}$ Oct. } & Balady & 0 & 2.349 & 2.265 & 1.728 & 1.200 & 4.614 & 6.342 & 7.542 & 48.83 \\
\hline & & $1 \mathrm{ml} / \mathrm{l}$ & 2.409 & 2.317 & 1.829 & 1.270 & 4.726 & 6.555 & 7.825 & 53.06 \\
\hline & & $2 \mathrm{ml} / \mathrm{l}$ & 2.590 & 2.369 & 1.937 & 1.340 & 4.959 & 6.896 & 8.236 & 54.89 \\
\hline & Sids 40 & 0 & 2.071 & 2.015 & 1.458 & 0.930 & 4.086 & 5.544 & 6.474 & 48.46 \\
\hline & & $1 \mathrm{ml} / \mathbf{l}$ & 2.133 & 2.040 & 1.551 & 1.010 & 4.173 & 5.724 & 6.734 & 52.50 \\
\hline & & $2 \mathrm{ml} / \mathrm{l}$ & 2.313 & 2.092 & 1.661 & 1.070 & 4.405 & 6.066 & 7.136 & 54.66 \\
\hline \multirow[t]{6}{*}{$15^{\text {th }}$ Oct. } & Balady & 0 & 2.195 & 1.782 & 1.535 & 0.930 & 3.977 & 5.512 & 6.442 & 43.87 \\
\hline & & $1 \mathrm{ml} / \mathbf{l}$ & 2.309 & 1.933 & 1.703 & 1.040 & 4.242 & 5.945 & 6.985 & 47.09 \\
\hline & & $2 \mathrm{ml} / \mathrm{l}$ & 2.404 & 2.019 & 1.754 & 1.120 & 4.423 & 6.177 & 7.297 & 48.95 \\
\hline & Sids 40 & 0 & 1.919 & 1.513 & 1.258 & 0.650 & 3.432 & 4.690 & 5.340 & 43.21 \\
\hline & & $1 \mathrm{ml} / \mathbf{l}$ & 2.042 & 1.656 & 1.375 & 0.770 & 3.698 & 5.073 & 5.843 & 46.54 \\
\hline & & $2 \mathrm{ml} / \mathrm{l}$ & 2.095 & 1.748 & 1.494 & 0.850 & 3.843 & 5.337 & 6.187 & 48.74 \\
\hline \multicolumn{3}{|c|}{ LSD at 0.05 level } & 0.039 & 0.049 & 0.077 & 0.050 & 0.053 & 0.083 & 0.092 & 0.91 \\
\hline
\end{tabular}


Table 14. Effect of the triple interaction among planting date, cultivar and potassium silicate rate on yield and its components of garlic during 2017/2018 season

\begin{tabular}{|c|c|c|c|c|c|c|c|c|c|c|}
\hline \multirow[t]{2}{*}{ Treatment } & & & \multicolumn{7}{|c|}{ Yield and its components (ton/fad.) } & \multirow{2}{*}{$\begin{array}{c}\text { Average } \\
\text { bulb weight } \\
\text { (g) } \\
\end{array}$} \\
\hline & & & $\begin{array}{c}\text { Grade } \\
\text { (1) }\end{array}$ & $\begin{array}{c}\text { Grade } \\
(2)\end{array}$ & $\begin{array}{c}\text { Grade } \\
(3) \\
\end{array}$ & $\begin{array}{c}\text { Grade } \\
\text { (4) }\end{array}$ & $\begin{array}{c}\text { Exportable } \\
\text { yield }\end{array}$ & $\begin{array}{c}\text { Marketable } \\
\text { yield }\end{array}$ & $\begin{array}{l}\text { Total } \\
\text { yield } \\
\end{array}$ & \\
\hline \multicolumn{11}{|c|}{ Planting date Cultivar K silicate rate } \\
\hline \multirow[t]{6}{*}{$15^{\text {th }}$ Sep. } & Balady & $\mathbf{0}$ & 1.943 & 1.139 & 1.314 & 0.829 & 3.082 & 4.396 & 5.225 & 36.39 \\
\hline & & $1 \mathrm{ml} / \mathrm{l}$ & 2.149 & 1.351 & 1.434 & 0.936 & 3.500 & 4.934 & 5.870 & 39.00 \\
\hline & & $2 \mathrm{ml} / \mathrm{l}$ & 2.260 & 1.325 & 1.568 & 1.099 & 3.585 & 5.153 & 6.252 & 40.26 \\
\hline & Sids 40 & $\mathbf{0}$ & 1.666 & 0.862 & 1.037 & 0.551 & 2.528 & 3.565 & 4.116 & 36.55 \\
\hline & & $1 \mathrm{ml} / \mathrm{l}$ & 1.872 & 1.074 & 1.157 & 0.650 & 2.946 & 4.103 & 4.753 & 39.16 \\
\hline & & $2 \mathrm{ml} / \mathrm{l}$ & 1.991 & 1.131 & 1.298 & 0.822 & 3.122 & 4.420 & 5.242 & 40.36 \\
\hline \multirow[t]{6}{*}{$1^{\text {st }}$ Oct. } & Balady & $\mathbf{0}$ & 2.340 & 1.696 & 1.719 & 1.208 & 4.036 & 5.755 & 6.963 & 45.46 \\
\hline & & $1 \mathrm{ml} / \mathrm{l}$ & 2.416 & 1.811 & 1.826 & 1.276 & 4.227 & 6.053 & 7.329 & 47.10 \\
\hline & & $2 \mathrm{ml} / \mathrm{l}$ & 2.629 & 1.825 & 1.922 & 1.356 & 4.454 & 6.376 & 7.732 & 53.17 \\
\hline & Sids 40 & $\mathbf{0}$ & 2.064 & 1.428 & 1.451 & 0.930 & 3.492 & 4.943 & 5.873 & 45.66 \\
\hline & & $1 \mathrm{ml} / \mathrm{l}$ & 2.138 & 1.043 & 1.549 & 1.015 & 3.181 & 4.730 & 5.745 & 46.91 \\
\hline & & $2 \mathrm{ml} / 1$ & 2.351 & 1.16 & 1.657 & 1.078 & 3.511 & 5.168 & 6.246 & 53.27 \\
\hline \multirow[t]{6}{*}{$15^{\text {th }}$ Oct. } & Balady & $\mathbf{0}$ & 2.203 & 1.293 & 1.550 & 0.936 & 3.496 & 5.046 & 5.982 & 39.26 \\
\hline & & $1 \mathrm{ml} / \mathbf{l}$ & 2.307 & 1.429 & 1.703 & 1.067 & 3.736 & 5.439 & 6.506 & 42.56 \\
\hline & & $2 \mathrm{ml} / \mathrm{l}$ & 2.377 & 1.543 & 1.749 & 1.249 & 3.920 & 5.669 & 6.918 & 54.61 \\
\hline & Sids 40 & $\mathbf{0}$ & 1.926 & 1.017 & 1.274 & 0.658 & 2.943 & 4.217 & 4.875 & 39.35 \\
\hline & & $1 \mathrm{ml} / \mathrm{l}$ & 2.037 & 1.151 & 1.426 & 0.789 & 3.188 & 4.614 & 5.403 & 42.71 \\
\hline & & $2 \mathrm{ml} / \mathrm{l}$ & 2.100 & 1.265 & 1.462 & 0.971 & 3.365 & 4.827 & 5.798 & 44.75 \\
\hline \multicolumn{3}{|c|}{ LSD at 0.05 level } & 0.073 & 0.318 & 0.063 & 0.117 & 0.311 & 0.321 & 0.346 & 6.99 \\
\hline
\end{tabular}

planting Balady cultivar on $1^{\text {st }}$ Oct. and spraying with $\mathrm{K}$ silicate at $1 \mathrm{ml} / 1$ in the $2^{\text {nd }}$ season only.

\section{Conclusion}

From foregoing results it could be concluded that, planting Balady cultivar on $1^{\text {st }}$ Oct. and spraying with $\mathrm{K}$ silicate at $2 \mathrm{ml} / 1$ increased total dry weight and yield and its components as well as average bulb weight.

\section{REFERENCES}

Abdalla, M.A., M.H. Aboul-Nasr, A.K. Metwaly and H.A. Shreen (2011). Growth and yield of fifteen garlic ecotypes. Assiut J. Agric. Sci., 36 (5): 52-65.

Abdel-Razzak, H.S. and G.A. El-Sharkawy (2013). Effect of biofertilizer and humic acid applications on growth, yield, quality and storability of two garlic (Allium sativum L.) cultivars. Asian J. Crop Sci., 5: 48-64.

Abo El-Fadel, N.I. and W.H. Mohamed (2013). Response of two garlic cultivars to foliar nutrition under irrigation with saline water. Egypt. J. Soil Sci., 53 (2): 207-220.
Abou El-Khair, E.E. and A.A.M. Mohsen (2016). Effect of natural sources of potassium on growth, mineral uptake and productivity of Jerusalem artichoke grown in new reclaimed soil conditions. Middle East J. Agric. Res., 5 (3): 367-377.

Abou-Baker, A.H., M. Abd-Eladl and M.M. Abbas (2011). Use of silicate and different cultivation practices in alleviating salt stress effect on bean plants. Aust. J. Basic and Appl. Sci., 5 (9):769-781.

Ahmed, H.M. (2002). Studies on the effect of fertilization by chemical, organic and biofertilization on growth, yield and quality of garlic (Allium sativum L.) under reclaimed soil. Ph.D. Thesis, Fac. Agric., Assuit Univ., Egypt.

Bhuiya, M.A.K., M.A. Rahim and M.N.A. Choudhury (2003). Effect of planting time, mulch and irrigation on the growth and yield of garlic. Asian J. Plant Sci., 2 (8): 639-643.

Choudhary, K. (2015). Effect of sowing time and sulphur levels on growth yield and 
quality of garlic (Allium sativum L.). M.Sc. Thesis, Fac. Sri Karan Narendra Agric., Jobner Univ., Endia.

Elrys, A.S. and A.M.A. Merwad (2017). Effect of alternative spraying with silicate and licorice root extract on yield and nutrients uptake by pea plants. Egypt. J. Agron., 39 (3): $279-292$.

El-Zohiri, S.S.M. and A.A. Farag (2014). Relation planting date, cultivars and growing degree-days on growth, yield and quality of garlic. Mid. East J. Agric. Res., 3 (4): 1169 1183.

Epstein, E. (1994). The anomaly of silicon in plant biology. In: Proc Natl Acad Sci USA, 91, 11-17.

Epstein, E. (1999). Silicon. Plant Mol Biol, 50, 641- 664 .

FAO (2016). Food and Agriculture Organization of the United Nations.

Gunda, V. (2013). Effect of planting time and plant densities on growth, yield and quality of garlic (Allium sativum L.) cv. Jamnagar. M.Sc. Thesis, Coll. Hort., Rajendranagar Dr. Y.S.R. Hort. Univ., India.

Hassan G.H, M.A. El-Shal, KH.A. Soliman and, I.M Ghoneim (2016). Effect of planting dates and periods of curing on quantitative, qualitative characteristics and storage ability of two garlic cultivars. J. Agric. Res. Kafr ElSheikh Univ., 42 (3): 401-414.

Hassan, A.H. (2015). Improving growth and productivity of two garlic cultivars (Allium sativum L.) grown under sandy soil conditions. Mid. East J. Agric. Res., 4 (2): 332- 346.

Jones, H.A. and L.K. Mann (1963). Onions and Their Allies. Leonard Hill (Book) Limited, London, 286.

Kamal, A.M. (2013). Influence of irrigation levels, antitranspirants and potassium silicate on growth, fruit yield and quality of sweet pepper plants (Capsicum annuum L.) grown under drip irrigation. J. Plant Production, Mansoura Univ., 4 (11):1581 - 1597.
Kurian, J.C. (1995). Plant that Heal (1 Ed.). Orienta I Watchman Publishing House, Pune, India, 31.

Merwad, A.M.A. (2018). Effect of lithovit and chitosan treatments on the productivity and storability of garlic. M.Sc. Thesis, Fac. Agric., Zagazig Univ., Egypt.

Ministry of Economic for Garlic Exportation (1963). To control exported garlic. Ministerial order No.652.

Mohammad, I.A.M. (2018). Effect of planting dates and nitrogen fertilization sources on productivity and quality of garlic plants under South Sinai Governorate conditions. M.Sc. Thesis, Fac. Agric., Zagazig Univ., Egypt.

Mohsen, A.A.M. (2012). Response of garlic plant to nitrogen, phosphorus, potassium and some biofertilizer levels under sandy soil conditions. Ph.D. Thesis, Fac. Agric. Zagazig Univ., Egypt.

Muhammad, J., M. Ishtiaq, N. Naeem, N. Muhammad, B. Jamiher and J. Iqbal (2001). Effects of different planting dates and spacing on growth and yield of garlic cv. Bianco. On line J. Bio. Sci., 1 (4): 206-208.

Purseglove, J.W. (1975). Tropical Crops: Monocotyledons, ELBS Longman, London, $52-56$.

Qin, Z. and S.P. Tian (2009). Enhancement of bio control activity of Cryptococcus laurentii by silicon and the possible mechanisms involved. Phytopathol., 95:69- 75.

Rahim, M.A. and A. Fordham (1988). Effects of storage temperature on the initiation and development of garlic cloves (Allium sativum L.). Sci. Hort., 37 (1-2): 25-28.

Rahim, M.A., M.N.A. Chowdhury, H.R.M.M. Anwar and M.S. Alam (2003). Effect of planting dates on the growth and yield of garlic germplasm. Asian J. Plant Sci., 2 (2): 171-174.

Salim, B.B.M., H.G. Abd El-Gawad and A. Abou El-Yazied (2014). Effect of foliar spray of different potassium sources on growth, yield and mineral composition of potato (Solanum tuberosum L.). Mid. East J. Appl. Sci., 4 (4): 1197-1204. 
Snedecor, G.W. and W.G. Cochran (1980). Statistical Methods. $7^{\text {th }}$ Ed. Iowa State Univ. Press, Ames, Iowa, USA, 507.

Vidya, G., M. Padma and M. Rajkumar (2013). Effect of planting time and plant densities on yield, quality and cost of production in garlic (Allium sativum L.) cv. Jamnagar. Asian J. Hort., 8 (2): 552-555.
Wand, S.Y. and G.J. Galletta, (1998). Foliar application of potassium silicate induces metabolic changes in strawberry plants. J. Plant Nutr. 21(1):157-167.

Youssef, N.S. and H.S.H. Tony (2014). Influence of different planting dates on the performance of new garlic genotypes grown under ElMinia Governorate conditions. Nat. Sci., 12 (5): 112-119.

تأثيــر ميعـاد الـزراعـة والـرش الورقي بسليكات البوتاسيوم على نمو ومحصول بعض أصناف الثوم ياسر عبدالعزيز السيد محمد ـ المتولى عبدالسميع الغمرينى - عبدالله برديسى ـ داليا أحمد سامى نوار

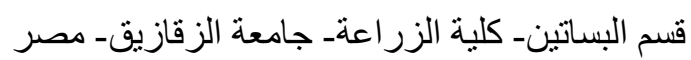

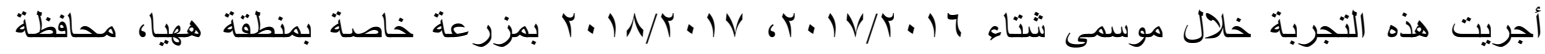

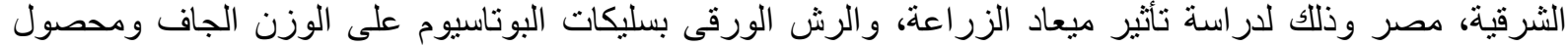

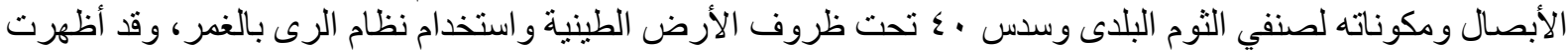

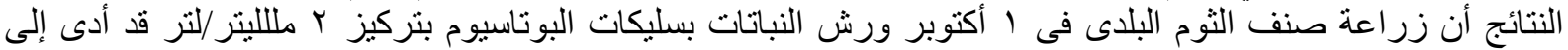

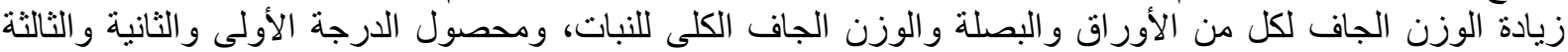

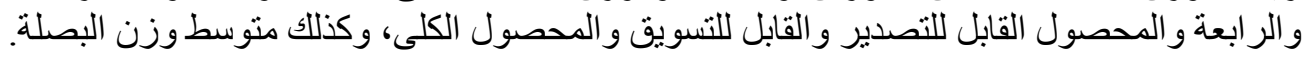

